\title{
INFLUENCIA DE LA HUMEDAD EN EL ENSAYO DE RESISTENCIA DE LOS LADRILLOS DE SUELO-CEMENTO
}

\author{
(INFLUENCE OF HUMIDITY ON THE STRENGTH TEST OF THE \\ SOIL-CEMENT BRICKS)
}

\author{
José I. Lima Díaz, ingeniero civil \\ Profesor de la Universidad de Camagüey. \\ Dunia Escobar Martín, ingeniero civil \\ E.C.I. nº 1. Ciego de Ávila. \\ CUBA.
}

Fecha de recepción: 27 - IX - 93

\section{RESUMEN}

A través de un diseño experimental adecuado se pretende mostrar la influencia de la humedad en el ensayo a compresión de los ladrillos de suelo-cemento, obteniendo las ecuaciones que muestran esta relación.

Su generalización posterior permitió elaborar una matriz de correlación de las resistencias con las diferentes humedades de ensayo, de forma tal que se pueda ensayar con una humedad dada y estimar la resistencia que tendrá esa muestra para diferentes valores de humedad, con un buen nivel de confianza. Esto posibilita que se puedan realizar comparaciones de resistencia entre elementos ensayados en condiciones desiguales de humedad, verificar el cumplimiento de los valores recomendados de resistencia y referirlos a un valor de humedad base, defecto actual de las Instrucciones Técnicas vigentes.
SUMMARY

The article seeks to show the influence of humidity on the compression test of the soil-cement bricks throngh an adapted experimental design. The equations showing this relation are obtained.

The subsequent generalization provides the possibility of making a pattern of correlation of strengths with different test humidities. Thus, the tests could be performed with a given humidity and the strength of the particular specimen for different humidity values could be estimated with a high degree of certainty. This makes possible the comparison of strength between tested elements in different humidity conditions, verification of fulfillment of recommended strength values and their expressing in terms of a basic humidity value, which is what the currently valid Technical Instructions lack.

\section{INTRODUCCIÓN}

Con el actual renacer de las técnicas de "bajo consumo" o "alternativas" como algunos prefieren llamar, tienen un papel destacado las producciones de suelo-cemento (ladrillos, bloques, machimbloques, bovedillas, tapial, etc).
El control de calidad de estos elementos está siendo efectuado mediante ensayos de laboratorio basados en parámetros no estándares (tipo de probetas, velocidad de rotura, humedad del material en el ensayo), debido a la falta de una Norma Cubana (NC) al respecto y de bibliografía de consulta en general. 
Además los documentos emitidos al abordar el control de calidad se tornan ambiguos al no especificar los parámetros de ensayo; no conocemos que ninguna Instrucción Técnica (IT) haya sido emitida para especificar cómo debe efectuarse el ensayo a compresión de los elementos de suelo-cemento, cuál debe ser la velocidad de rotura ni cuál la humedad del material a la hora del ensayo; aspecto ya tratado en el hormigón celular "1:2".

Por ejemplo la IT 07-91 plantea que la resistencia a compresión debe ser $2 \mathrm{MPa}$ como promedio y 1,7 MPa para valores individuales pero no especifica la humedad del ladrillo en el ensayo ${ }^{(3)}$, lo cual afecta sensiblemente al valor obtenido de resistencia a compresión del mismo, como se aprecia en la gráfica. 1. Referido a este último aspecto, algunos especialistas recomiendan ensayar saturado el material, otros plantean 4 horas de inmersión en agua como mínimo y otro grupo numeroso está ensayando sus producciones con la humedad que tenga en ese momento en la obra o en el lugar de almacenamiento, lo cual tiene gran variabilidad; por tanto, los datos aportados por ellos tienen poca base para hacer comparaciones, e incluso conocer si se cumple con un parámetro recomendado. Vale la pena destacar que con igual tiempo de inmersión en agua podrá lograrse igual saturación, pero no se logra igual humedad, producto de que esta última depende de la porosidad del material.

\section{MÉTODO EXPERIMENTAL}

Tratar de conocer cómo influye en la resistencia la humedad en elementos de suelo-cemento fue nuestro objetivo en una investigación realizada con ladrillos elaborados en la Empresa de Construcciones Varias del Poder Popular en Morón. Éstos estaban elaborados con suelo de un préstamo conocido por Guanito (clasificación: SUCS $=$ ML y $\mathrm{HBR}=\mathrm{A}-4^{(3)}$ ) y cemento P-350 de Siguaney; elaborados con las siguientes relaciones en volumen: 1:10; $1: 12 ; 1: 14 ; 1: 16 ; 1: 18 ; 1: 20$.

Conociendo que la resistencia a compresión estaba afectada por los siguientes factores fundamentales:

\section{1.- Humedad \\ 2.- Densidad \\ 3.- Dosificación}

y que la humedad del ladrillo en condiciones de (c) Consejo Superior de Investigaciones Científicas Licencia Creative Commons 3.0 España (by-nc) obra estatia culce 11\% y 19\%, relacionando las resistencias obtenidas y la resistencia para el $15 \%$ de humedad en cada dosificación, obtuvimos la relación que muestra la tabla 1 y la gráfica 1 . De esta forma eliminamos el factor dosificación.

\section{Gráficà 1}

Relación humedad vs resistencia para el suelo-cemento

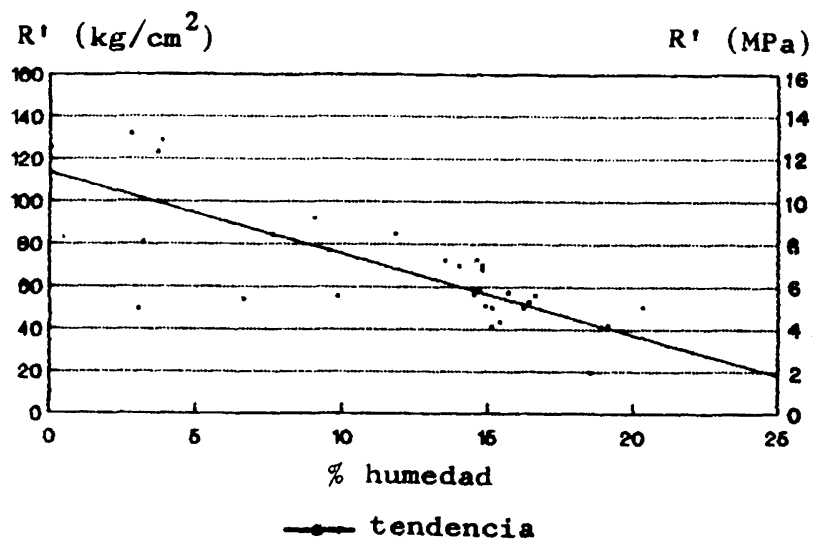

Fuente: Lab. Morón ECI ne 1

Tabla 1

\begin{tabular}{|c|c|c|c|c|c|}
\hline Dos & $\mathrm{h} \approx 15 \%$ & $\mathrm{R}^{\prime} 15 \mathrm{~h}$ & $\%$ hum & $\mathrm{R}^{\prime} \mathrm{i}$ & $\mathrm{R} 15 / \mathrm{Ri}$ \\
\hline 10 & 14,55 & 70 & 0 & 125 & 0,56 \\
12 & 15,83 & 54 & 3,7 & 123 & 0,44 \\
12 & 15,83 & 54 & 9,5 & 77 & 0,70 \\
14 & 14,60 & 65 & 9 & 92 & 0,71 \\
14 & 14,60 & 65 & 3,8 & 129 & 0,50 \\
14 & 14,60 & 65 & 11,8 & 85 & 0,76 \\
16 & 15,27 & 53 & 2,8 & 132 & 0,40 \\
16 & 15,27 & 53 & 7,6 & 84 & 0,63 \\
18 & 15,40 & 43 & 19,3 & 37 & 1,16 \\
18 & 15,40 & 43 & 3,2 & 81 & 0,53 \\
20 & 15,10 & 45 & 6,6 & 54 & 0,83 \\
20 & 15,10 & 45 & 9,8 & 55 & 0,82 \\
\hline
\end{tabular}

Donde:

Dos : Relación de suelo de la dosificación.

$h \approx 15 \%$ : Promedio de los porcentaje de humedad cercanos al $15 \%$.

R'15h : Promedio de las resistencias cercanas al $15 \%$.

\%Hum : Porcentaje de humedad del material a la hora del ensayo.

$\mathrm{R}^{\prime} \mathrm{i}$ : Resistencia a compresión de los ladrillos para diferentes humedades.

R15h/Ri : Relación entre R'15h y R'i.

La recta mínimo cuadrática ajustada, tomando al 15\% de humedad cómo patrón, sería la siguiente: 
$\mathrm{R}^{\prime} 15 \mathrm{~h} / \mathrm{R}^{\prime} \mathrm{i}=0,46+0,036 \%$ hum

por tanto:

$R^{\prime} 15 h=R^{\prime} i(0,46+0,036 \%$ hum $)$

llamándole coeficiente de correlación (coef) a:

coef $=0,46+0,036$ hum

quedando la ecuación I de la siguiente forma:

$R^{\prime} 15=R^{\prime} i^{*}$ coef

que se muestra en la gráfica 2, y su generalización posterior en forma de matriz de correlación de las Resistencias en función de la humedad.

Gráfica 2

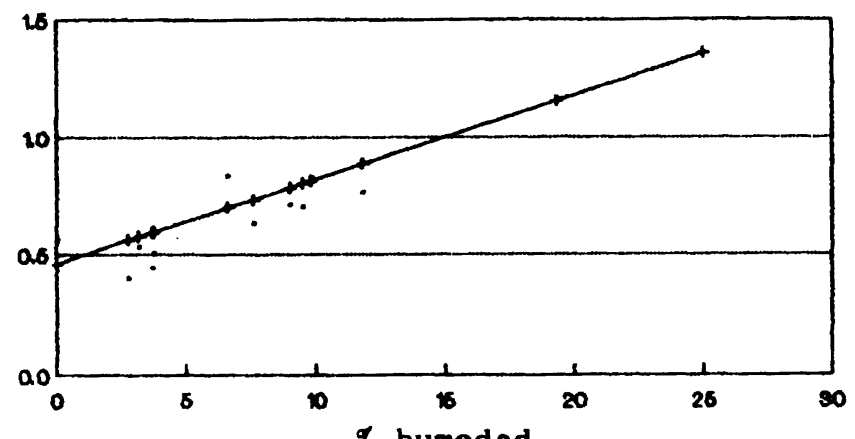

\% humedad

- R' 15\%hum/R'i

+ Coef $=0,46+0,036$ ghum

Fuente: Lab. Morón ECI ne 1

Matriz de correlación de los coeficientes de la Resistencia:

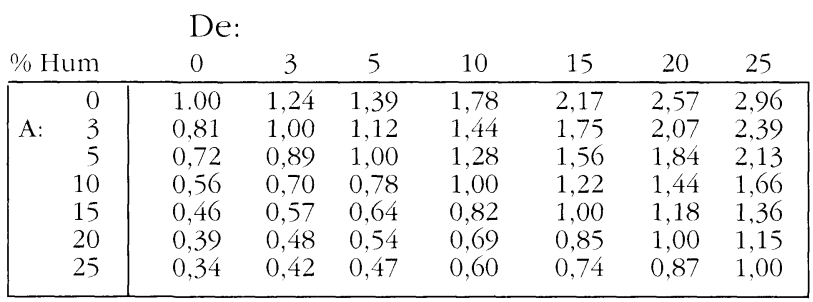

Ejemplo:

Si se obtuvo una $\mathrm{R}=2,8 \mathrm{MPa}$ con una humedad del $20 \%$. Se estimó la resistencia seca del material (\% hum $)=0$ :

$$
\begin{aligned}
\text { R0\%h } & =\text { Coef. } * 20 \% \mathrm{~h} \\
& =2,57 * 2,8 \\
& =7,2 \mathrm{MPa}
\end{aligned}
$$

Gorchakov"1, para el hormigón celular, plantea afectar a la resistencia por el coeficiente $\mathrm{kw}$ siguiente:

\begin{tabular}{|lccccccc|}
\hline Humedad en masa (\%) & 0 & 5 & 10 & 15 & 20 & $25 \mathrm{y}+$ \\
\hline valor de kw & 0,8 & 0,9 & 1,0 & 1,05 & 1,10 & 1,15 \\
\hline "Coef. p/ 15\% hum. & 0.76 & 0,86 & 0,95 & 1,0 & 1,05 & 1,10 \\
\hline
\end{tabular}

$$
\text { * valores calculados. }
$$

y Makaritchev ${ }^{(3)}$ formuló la siguiente relación entre el hormigón húmedo (Rh) y seco (Rs):

\begin{tabular}{|ccccccc|}
\hline Humedad en peso (\%) & 0 & 5 & 10 & 15 & 20 & $25 \mathrm{y}+$ \\
\hline Rh/Rs & 1 & 0,9 & 0,8 & 0,75 & 0,70 & 0,65 \\
\hline Rh/Rs p/15\% hum. & 0,75 & 0,83 & 0,94 & 1,0 & 1,07 & 1,15 \\
\hline
\end{tabular}

"valores calculados.

Comparando los valores calculados para el hormigón celular con los obtenidos para el suelo-cemento (fila 5 de la matriz) podemos darnos cuenta que los elementos de suelo-cemento están más afectados por la bumedad durante el ensayo de Resistencia a Compresión.

\section{RESULTADOS}

Mediante el empleo de los coeficientes anteriores

\begin{tabular}{|c|c|c|c|c|c|c|c|c|}
\hline DOS & \%hum & & & & $\underline{R^{\prime} i}$ & & Ncon & \\
\hline 10 & $\begin{array}{r}0,00 \\
14,60 \\
14,80 \\
14,80 \\
14.00\end{array}$ & $\begin{array}{l}1.855 \\
1.754 \\
1.707 \\
1.815 \\
1.800\end{array}$ & $\begin{array}{l}\bar{X}= \\
S= \\
\delta=\end{array}$ & $\begin{array}{l}6,06 \\
1,17 \\
2,87\end{array}$ & $\begin{array}{ll}0 & \\
8 & x= \\
0 & 5= \\
2 & 8= \\
5 & \end{array}$ & & $\begin{array}{l}57,55 \\
71,44 \quad \bar{x}= \\
69,29 \mathrm{~S}= \\
67,23 \quad \delta= \\
67,05\end{array}$ & $\begin{array}{r}66,51 \\
4,76 \\
7,15\end{array}$ \\
\hline $\begin{array}{l}12 \\
12 \\
12 \\
12 \\
12\end{array}$ & $\begin{array}{r}3,70 \\
14,70 \\
16,20 \\
9,50 \\
16,60\end{array}$ & $\begin{array}{l}1.795 \\
1.777 \\
1.830 \\
1.779\end{array}$ & $\begin{array}{l}\bar{x}= \\
S= \\
\delta=\end{array}$ & $\begin{array}{l}44 \\
29 \\
62\end{array}$ & $\begin{array}{c}122,55 \\
57,30 \quad \bar{x}= \\
49,46 \quad \mathrm{~S}= \\
76,74 \quad \delta= \\
55,35\end{array}$ & & $\begin{array}{ll}72,70 & \\
56,68 & \bar{x}= \\
51,60 & \mathrm{~S}= \\
61,55 \quad \delta= \\
58,54\end{array}$ & $\begin{array}{r}7.03 \\
11.68\end{array}$ \\
\hline $\begin{array}{l}14 \\
14\end{array}$ & $\begin{array}{r}9,00 \\
3,80 \\
15,70 \\
11,80 \\
13,50\end{array}$ & $\begin{array}{l}1.861 \\
1.817 \\
1.843 \\
1.837\end{array}$ & $\begin{array}{l}\bar{x}= \\
S= \\
\delta=\end{array}$ & $\begin{array}{r}14 \\
0\end{array}$ & $\begin{array}{r}91,87 \\
128,69 \mathrm{x}= \\
57,12 \mathrm{~S}= \\
84,71 \delta= \\
71,84\end{array}$ & & $\begin{array}{ll}72,02 & \\
76,80 & \mathrm{x}= \\
58,56 \quad \mathrm{~S}= \\
74,95 \quad \delta= \\
67,96\end{array}$ & $\begin{array}{r}70,06 \\
6,48 \\
9,25\end{array}$ \\
\hline 10 & $\begin{array}{r}2,80 \\
7,60 \\
14,50\end{array}$ & $\begin{array}{l}1.783 \\
1.850 \\
1.834 \\
1.786\end{array}$ & $\begin{array}{l}\bar{x}= \\
S= \\
\delta=\end{array}$ & $\begin{array}{r}806,01 \\
29,92 \\
1,66\end{array}$ & $\begin{array}{r}52,50 \\
50,48 \mathrm{x}= \\
131,85 \mathrm{~S}= \\
84,31 \delta= \\
55,89\end{array}$ & & $\begin{array}{l}55,15 \\
50,30 \quad \overline{\mathrm{X}}= \\
73,94 \mathrm{~S}= \\
61.85 \mathrm{\delta}= \\
54,89\end{array}$ & $\begin{array}{r}8,23 \\
13,89\end{array}$ \\
\hline 10 & $\begin{array}{r}18,50 \\
19,10 \\
3,20\end{array}$ & $\begin{array}{l}1.764 \\
1.662 \\
1.602 \\
1.806\end{array}$ & $\begin{array}{l}\bar{x}= \\
S= \\
\delta=\end{array}$ & $\begin{array}{r}1.709,86 \\
72,30 \\
4,23\end{array}$ & $\begin{array}{l}49,88 \\
43,14 \mathrm{x}= \\
19,29 \mathrm{~S}= \\
41,48 \delta \mathrm{\delta}= \\
80,61\end{array}$ & & $\begin{array}{l}59,39 \\
43,77 \quad \bar{x}= \\
21,72 \quad \mathrm{~S}= \\
47,61 \quad \delta= \\
46,37\end{array}$ & $\begin{array}{l}12,26 \\
28,02\end{array}$ \\
\hline 20 & $\begin{array}{r}15,10 \\
6,60 \\
\end{array}$ & $\begin{array}{l}1.758 \\
1.770 \\
1.787 \\
\end{array}$ & $\begin{array}{l}\bar{x}= \\
S= \\
\delta=\end{array}$ & $\begin{array}{r}40 \\
2\end{array}$ & $\begin{array}{l}55,07 \mathrm{X}= \\
41,03 \mathrm{~S}= \\
49,35 \mathrm{\delta}= \\
53,79\end{array}$ & 9,90 & $\begin{array}{r}27,96 \\
4.76 \quad \bar{x}= \\
41,18 \quad S= \\
49,52 \quad \delta= \\
37,52\end{array}$ & $\begin{array}{r}40.19 \\
7.29 \\
18,13\end{array}$ \\
\hline
\end{tabular}
procesamos la resistencia obtenida ( $\left.R^{\prime} i\right)$ para las distintas humedades, y logramos adecuarlas a una

Tabla 2 
humedad patrón (15\%), lo que permitió una mayor claridad en el análisis. Nótese cómo en la tabla 2 se aprecian mucho más claramente, en los valores corregidos, las relaciones resistencia vs dosificación y resistencia vs densidad; así como la disminución de la dispersión de los valores de resistencia.

Donde, según la tabla 2:

Dos : Relación de suelo de la dosificación.

$\%$ Hum : Por ciento de humedad del material a la hora del ensayo.

Dseca : Densidad seca.

R’i : Resistencia a compresión de los ladrillos para diferentes humedades.

RcorR : Resistencia estimada para el 15\% de humedad según la recta mínimo cuadrática ajustada.

$\mathrm{x}$ : Valor medio correspondiente a la dosificación.

S : Desviación estándar de los valores de una misma dosificación.

$\delta$ : Coeficiente de variación de los anteriores.

La tabla 3 muestra una estimación de resistencia para cada valor de humedad.

Tabla 3

\begin{tabular}{|c|c|c|c|c|c|c|c|c|}
\hline$\underline{D O S}$ & \%hum & Dseca & $\underline{R^{\prime} i}$ & $\underline{\mathrm{R} \text { corR }}$ & RRest & $\mathrm{ii}^{*}$ & \%Rteo & \%Rreal \\
\hline 10 & 0.00 & 1.855 & 125,10 & 57.55 & 144.59 & 2.63 & 251.26 & 217.39 \\
\hline 10 & 14,60 & 1.754 & 72,48 & 71,44 & 67,48 & 0,37 & 94,46 & 101,46 \\
\hline 10 & 14,80 & 1.707 & 69,80 & 69,29 & 66,99 & 0,12 & 96,68 & 100.73 \\
\hline 10 & 14,80 & 1.815 & 67.72 & 67.23 & 66,99 & 0.01 & 99.65 & 100,73 \\
\hline 10 & 14,00 & 1.800 & 69,55 & 67,05 & 69,00 & 0,00 & 102,90 & 103,73 \\
\hline 12 & 3,70 & 1.851 & 122,55 & 72,70 & 101.50 & 4,36 & 139,63 & 168,58 \\
\hline 12 & 14.70 & 1.795 & 57,30 & 56,68 & 60,87 & 0,21 & 107,39 & 101,09 \\
\hline 12 & 16,20 & 1.777 & 49,46 & 51,60 & 57,72 & 1,18 & 111,86 & 95.86 \\
\hline 12 & 9.50 & 1.830 & 76,74 & 61,55 & 75.08 & 0,04 & 121,99 & 124,69 \\
\hline 12 & 16,60 & 1.779 & 55.35 & 58,54 & 56,93 & 0,04 & 97,26 & 94.55 \\
\hline 14 & 9,00 & 1.832 & 91.87 & 72,02 & 89.36 & 0,07 & 124,07 & 127,55 \\
\hline 14 & 3.80 & 1.861 & 128.69 & 76,80 & 117.39 & 1.09 & 152,85 & 167,56 \\
\hline 14 & 15,70 & 1.817 & 57,12 & 58,56 & 68,34 & 1,84 & 116,70 & 97.54 \\
\hline 14 & 11,80 & 1.843 & 84,71 & 74,95 & 79,18 & 0.39 & 105,64 & 113,02 \\
\hline 14 & 13,50 & 1.837 & 71.84 & 67.96 & 74,06 & 0.07 & 108.98 & 105,71 \\
\hline 16 & 16,40 & 1.777 & 52,50 & 55,15 & 56,38 & 0,27 & 102,24 & 95,20 \\
\hline 16 & 14,90 & 1.783 & 50,48 & 50,30 & 59,44 & 1.35 & 118,16 & 100,36 \\
\hline 16 & 2,80 & 1.850 & 131.85 & 73,94 & 105.61 & 6.52 & 142,83 & 178.32 \\
\hline 16 & 7,60 & 1.834 & 84,31 & 61,85 & 80,73 & 0.16 & 130,54 & 136,31 \\
\hline 16 & 14,50 & 1.786 & 55,89 & 54,89 & 60,31 & 0,32 & 109,88 & 101,83 \\
\hline 18 & 20,30 & 1.715 & 49,88 & 59.39 & 36,76 & 4,68 & 61.89 & 83,98 \\
\hline 18 & 15,40 & 1.764 & 43,14 & 43,77 & 43,15 & 0,00 & 98,59 & 98,58 \\
\hline 18 & 18,50 & 1.662 & 19,29 & 21,72 & 38,87 & 9,87 & 179,00 & 88,81 \\
\hline 18 & 19,10 & 1.602 & 41,48 & 47,61 & 38,14 & 0.29 & 80,12 & 87,14 \\
\hline 18 & 3,20 & 1.806 & 80,61 & 46,37 & 76,09 & 0.27 & 164,12 & 173.85 \\
\hline 20 & 3,00 & 1.695 & 49.23 & 27.96 & 70.76 & 6,55 & 253,03 & 176,06 \\
\hline 20 & 9,80 & 1.818 & 55.07 & 44,76 & 49,45 & 0,64 & 110,46 & 123,03 \\
\hline 20 & 15,10 & 1.758 & 41.03 & 41,18 & 40,04 & 0,02 & 97,25 & 99,64 \\
\hline 20 & 15,10 & 1.770 & 49.35 & 49,52 & 40,04 & 2,16 & 80.86 & 99.64 \\
\hline \multirow[t]{2}{*}{20} & 6,60 & 1.787 & 53.79 & 37.52 & 57,61 & 0.25 & 153,54 & 143,35 \\
\hline & & & & & suma & 45,77 & & \\
\hline
\end{tabular}

Donde:

Dos, \%hum, Dseca, R’i y RcorR ya fueron definidas para la tabla anterior.

RRest : R'15h/coef.

$\mathrm{ji}^{2}$ : Prueba de ji cuadrado= (observado-estimado $)^{2} /$ estimado.

\%Rteo : Relación porcentual entre cada valor de RRest y RcorR.

\%Rreal : Relación porcentual entre cada valor de R'i y RcorR.

Como ji² $(45,77)$ es menor que $\mathrm{ji}^{2} .99(48,3)$ no podemos rechazar el ajuste realizado con un $99 \%$ de confianza.

La estimación a través de la recta fue más precisa que la realizada mediante el polinomio de segundo grado; por tanto, la matriz de correlación fue elaborada según la recta mínimo cuadrática.

Si tomamos como $100 \%$ de resistencia el valor correspondiente a $15 \%$ de humedad, la relación entre $\%$ de resistencia y $\%$ de humedad (que aparecen en las dos últimas columnas) quedaría como muestra la gráfica 3:

Gráfica 3

Relación porcentual humedad vs resistencia

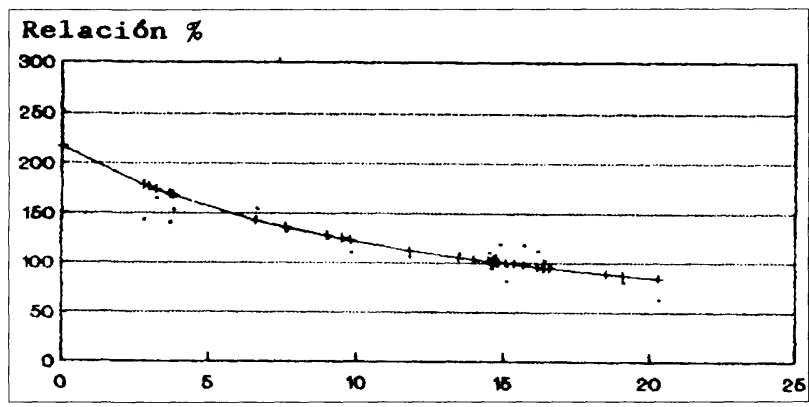

$\%$ Rteo $=191-5,72 \%$ hum

$\%$ Rreal $=191-5,98 \%$ hum

que muestran más claramente la influencia de la humedad del suelo-cemento a la hora del ensayo. 


\section{CONCLUSIONES}

- Se debe siempre que se ensayen a compresión elementos de suelo-cemento, determinar la humedad del mismo y referirla a un valor base, por ejemplo: el 15\% de humedad.

- Recomendamos ensayar los elementos con una humedad próxima a la de saturación, debido a que en su estado seco se producen en ocasiones destrucciones locales dentro del mismo, sin alcanzar su resistencia final; lo cual se elimina con la lubricación que provoca la humedad entre las partículas del suelo-cemento.

- Cuando aparezca un valor de resistencia recomendado para el suelo-cemento, debe referirse a su contenido de humedad a la hora del ensayo.
- Se recomienda el empleo de la matriz de correlación de la resistencia vs diferentes humedades para realizar comparaciones referidas a una humedad base.
NOTAS

(1) Gorchakov, G.I.: Materiales de Construcción, Editorial MIR, URSS, 1984

(2) Lewicki, B.: Edificios de viviendas prefabricadas con elementos de grandes dimensiones, Editorial Arkady, Polonia, 1968.

(3) Instrucción Técnica IT-07-91, "Ladrillos macizos de suelocemento", La Habana, Cuba, junio 1991.

\section{publicaciones del ICCET/CSIC}

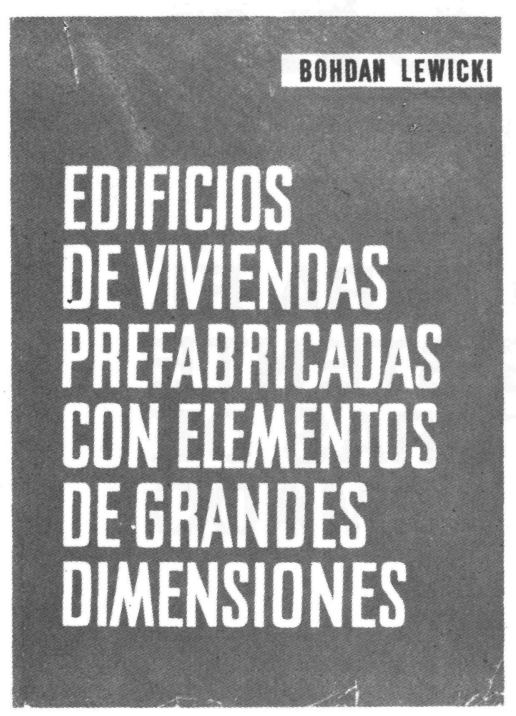

Bohdan Lewicki

Este libro trata de los problemas relativos a la construcción de los edificios de viviendas o publicos realizados con elementos prefabricados de grandes dimensiones. Se han estudiado los problemas de arriostramiento, asi como los que plantea la resistencia de los elementos y de la estructura; se han examinado las cuestiones de orden higrotérmico, acústico y de resistencia al fuego; también se ha profundizado en el estudio de la estanquidad de los muros exteriores y de las juntas.

La obra incluye numerosas ilustraciones que dan detalles de diversas soluciones, as como ejemplos de cálculo, tablas de valores numéricos, diagramas y ábacos.

Un volumen encuadernado en tela, de $24 \times 17 \mathrm{~cm}$, compuesto de 616 págs.

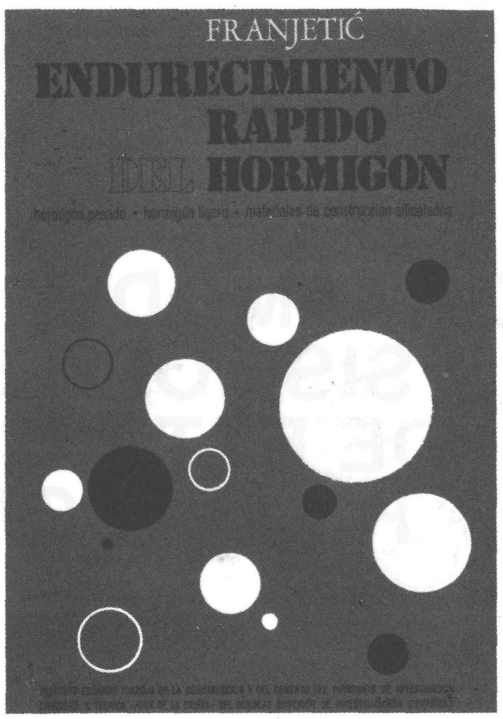

\section{Zorislav Franjetić}

En la obra de Franjetić se expone de una forma minuciosa, ordenada y sistemática, todo un cuerpo de doctrina que reúne el conocimiento actual sobre el endurecimiento rápido del hormigón. Parte el autor de los principios básicos y llega a las últimas consecuencias y realidades técnicas y economicas.

Es una obra de consulta, tanto para el investigador sobre la materia, como para el proyectista y el realizador y montador de plantas e instalaciones y equipos de curado y endurecimiento rápido.

Un volumen encuadernado en cartóné, de $17 \times 24,5 \mathrm{~cm}$, compuesto de 385 págs. 110 figuras y 10 tablas.

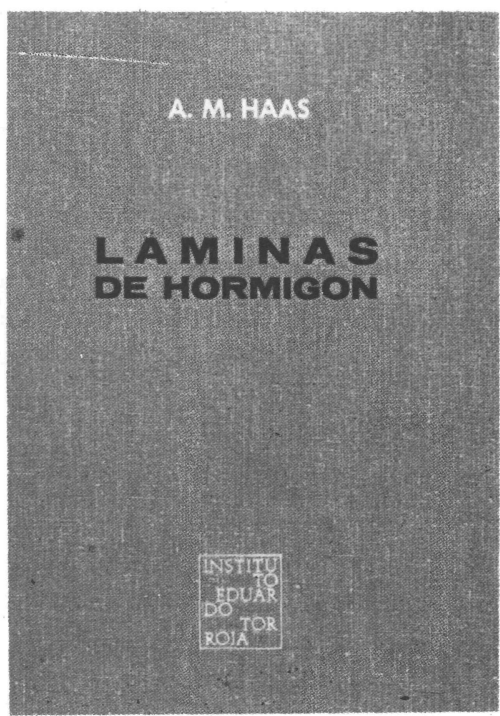

\section{A. M. Haas}

Al escribir este libro el autor intento poner a disposición de los estudiantes y de los ingenieros unos conocimientos prácticos, adecuados para servir de guia en el diseño y construcción de láminas delgadas de hormigón.

El autor está convencido de que el éxito en el diseño de una lámina exige, por parte del proyectista, un examen de las tres fases por las que pasa la materialización de la lámina: las que pasa la materialización de la lámina:
el diseñc, el análisis estructural y la construcción de la estructura.

Un volumen encuadernado en tela, de $17 \times 24,5 \mathrm{~cm}$, compuesto de 420 págs., 141 figuras, 22 fotografias y 6 tablas. 